\title{
Reflets
}

Revue d'intervention sociale et communautaire

\section{Pratiques d'intervention féministe auprès des femmes survivantes d'agression à caractère sexuel : le contexte franco-ontarien}

\section{Cécile Coderre et Joanne Hart}

Volume 9, numéro 1, printemps 2003

Le genre en contexte : pratiques sociales et représentations

URI : https://id.erudit.org/iderudit/010867ar

DOI : https://doi.org/10.7202/010867ar

Aller au sommaire du numéro

Éditeur(s)

Reflets : Revue ontaroise d'intervention sociale et communautaire

ISSN

1203-4576 (imprimé)

1712-8498 (numérique)

Découvrir la revue

Citer cet article

Coderre, C. \& Hart, J. (2003). Pratiques d'intervention féministe auprès des

femmes survivantes d'agression à caractère sexuel : le contexte

franco-ontarien. Reflets, 9(1), 186-210. https://doi.org/10.7202/010867ar

Tous droits réservés (C) Reflets : Revue ontaroise d'intervention sociale et communautaire, 2002
Ce document est protégé par la loi sur le droit d'auteur. L'utilisation des services d'Érudit (y compris la reproduction) est assujettie à sa politique d'utilisation que vous pouvez consulter en ligne.

https://apropos.erudit.org/fr/usagers/politique-dutilisation/ 


\section{Pratiques d'intervention féministe auprès des femmes survivantes d'agression à caractère sexuel : le contexte franco-ontarien}

Cécile Coderre et Joanne Hart ${ }^{1}$

Université d'Ottawa

Presque trente ans ont passé depuis la parution des premiers textes sur l'intervention féministe, dix ans depuis la tenue du premier colloque sur l'intervention féministe en Ontario et sept ans depuis le premier colloque franco-ontarien sur l'agression à caractère sexuel. De tels délais commandent un regard renouvelé sur les modèles d'intervention féministe. Qu'est-il advenu de ces modèles d'intervention? Comment ont-ils évolué? Les intervenantes féministes ont-elles défini des pratiques d'intervention spécifiques au contexte d'intervention minoritaire? Et quels sont les défis auxquels elles sont confrontées? C'est à ces questions que nous tenterons brièvement de répondre, en les appliquant plus particulièrement, mais non exclusivement, aux interventions liées à l'agression à caractère sexuel.

Après avoir présenté quelques définitions ainsi que les fondements de l'intervention féministe dans les situations d'agression sexuelle, nous analyserons certains modèles de pratique présents en Ontario français. En effet, la problématique de l'agression sexuelle est l'une des premières thématiques à avoir regroupé les militantes féministes de la première heure dans l'Est ontarien. Ce texte cherche ainsi à franchir les frontières de la 
culture orale et à mieux documenter les pratiques d'intervention. Car, comme le soulignaient (déjà en 1992) Maria De Koninck et Sylvie Savard, pour le contexte québécois :

La culture de l'intervention féministe est une culture orale et à l'instar de l'histoire des femmes, l'histoire de l'intervention n'est pas écrite. La mémoire collective se perd. Déjà certains lieux où ont pris forme des interventions originales n'existent plus et les informations nécessaires à la compréhension des démarches qui y ont été réalisées n'ont pas été colligées (De Koninck et Savard 1992 : 58).

Dans ce but, nous avons eu recours à des sources d'information diversifiées. Nous avons dépouillé des documents produits par divers organismes dont la mission première est d'offrir des services aux femmes victimes d'agression sexuelle. Nous nous sommes aussi appuyées sur des entrevues de groupe auprès de six (6) intervenantes féministes oeuvrant au sein d'organismes communautaires et institutionnels de l'Est ontarien, réalisées par une des auteures dans le cadre de son mémoire ${ }^{2}$. Toutefois, nous ne prétendons pas documenter l'ensemble de la réalité des pratiques en Ontario français; notre intention est plutôt de jeter les premières bases pour faciliter leur analyse.

En effet, cette recherche vise à dépasser la simple description pour s'inscrire dans une démarche de consolidation de l'intervention féministe. Car, comme De Koninck et Savard (1992) notaient que la recherche théorique sur l'intervention féministe était trop souvent absente, sans vouloir l'enfermer dans " une orthodoxie ", il est essentiel de démontrer son évolution, de la situer dans sa mouvance et dans cette dynamique qui fait son originalité, le lien théorie/pratique. Il s'agit donc de « dépasser le descriptif et de lui donner les assises nécessaires à sa poursuite et à son raffinement " (p. 60), de dépasser le côté technique de l'intervention féministe pour la situer dans une perspective politique, celle de la dynamique de la transformation des rapports sociaux de sexe. Et comme Helen Levine (1989, in de Koninck et Savard (1992)), le souligne : 
Le mandat de l'intervention féministe (feminist counselling) inclut un processus de guérison (healing process), un processus d'éducation (éducational process) et un processus politique (political process) basé sur une analyse féministe des rapports sociaux de sexe. (...). C'est une voie qui sert de liens entre les luttes des femmes dans un contexte politique à la fois comme offreuses de service et utilisatrices de service et travaillant ensemble en vue d'un changement. (trad libre).

Mais tout d'abord, situons brièvement les origines des approches féministes d'intervention.

\section{Thérapie féministe, intervention féministe et approche féministe d'intervention}

Dès leur origine, dans les années soixante-dix, les modèles féministes d'intervention s'inspirent de trois lieux d'ancrage : les critiques, souvent universitaires du sexisme en psychothérapie, l'expérience des groupes féministes de réflexion aussi nommés consciousness-raising groups et, enfin, l'intervention radicale, ellemême fille du courant critique de l'anti-psychiatrie.

\section{Aux origines : le mouvement des femmes}

Dans la mouvance de la renaissance du mouvement des femmes dans les années soixante-dix, des spécialistes s'interrogent sur la pertinence de la psychothérapie traditionnelle auprès des femmes. La multidisciplinarité est à l'ordre du jour. Psychologues, psychiatres et sociologues inscrits dans divers milieux de recherche et d'intervention aux États-Unis publient une somme intitulée Women in Therapy (Franks et Burtle 1974). Ils ouvrent de nouvelles pistes d'intervention sur des thèmes aussi diversifiés que l'alcoolisme, l'homosexualité féminine, le divorce, la santé mentale, et 
jettent un regard critique sur plusieurs approches traditionnelles que ce soient la thérapie cognitive, la gestalt ou encore la théorie freudienne. La thérapie féministe, terme utilisé alors aux États-Unis, se définit donc comme une forme alternative pour les femmes au sexisme en thérapie.

Dans le même temps, en marge de la thérapie professionnelle, se développent des groupes d'auto-conscientisation (consciousness-raising groups) (Kirsh 1974; Castro 1984). Plus connus aux États-Unis, ces petits groupes ont généralement un caractère informel et assez hétérogène. Quoique très différents les uns des autres, ils ont en commun la remise en question de la hiérarchie et le désir d'un mode de fonctionnement égalitaire. Ce sont aussi des lieux d'expression, des moments forts pour partager, avec les autres femmes, des expériences personnelles. Enfin, chaque femme y est considérée comme une autorité pour parler de son expérience (Kirsh 1974 : 343). En fait, on retrouve, dans ces premiers groupes,l'essence même des fondements de l'intervention féministe : démocratie sororale, expertise personnelle et analyse sociale.

Toutefois, ces groupes ne se définissent pas comme des groupes thérapeutiques. Ils s'inscrivent plutôt en faux contre cet aspect, considérant même la thérapie comme une forme de contrôle social des femmes (Morgan 1970; Chesler 1971 in Kirsh 1974 : 336-337). À cet égard, l'utilisation politique de l'expérience personnelle s'inscrit dans un autre courant de pensée critique, celui de l'anti-psychiatrie (Laing 1971; 1967) d'où naîtra un modèle radical d'intervention auprès des femmes appelé thérapie radicale. Cette dernière "privilégie " aussi le groupe comme moyen de combattre l'oppression. "La thérapie radicale incorpore certaines pratiques d'approches humanistes et s'inspire avant tout de l'analyse transactionnelle " (Tremblay et Tremblay $1996: 40)^{3}$.

C'est toutefois le terme intervention féministe qui obtient la cote de popularité dans les index universitaires (Bourgon 1987; Bourgon et Corbeil 1990; Corbeil et al. 1983; Doré 1982; Gaston et Hodgins 1984; Larouche 1993; Martin-Beausoleil 1982; PaquetDeehy et al 1992) ${ }^{4}$. Et malgré des origines aussi diversifiées, une des caractéristiques de l'intervention féministe est d'avoir réussi à arrimer le monde du savoir universitaire et celui du monde 
communautaire dans une démarche commune de réflexion sur l'intervention.

Ainsi, l'intervention féministe s'est développée autour de quatre principes : établir des rapports les plus égalitaires possible; faire connaitre la réalité des femmes; $s$ 'engager auprès des femmes; croire en leur force, en leur potentiel, en elles.

\section{Des ressources et des services, espaces de développement en Ontario français}

La décennie 70 est marquée par une lutte sans précédent au Canada comme aux États-Unis en matière de violence faite aux femmes, en matière de viol et d'agression à caractère sexuel (Côté 1995; Lamoureux 1998; 1997; Levan 1996; URSEL 1994). Les premiers centres d'aide aux victimes d'agression sexuelle à être créés sont marqués, à l'origine par leur inclusion dans les luttes des premiers services féministes offerts aux femmes qui en sont victimes. Dans plusieurs régions ontariennes, ces services étaient offerts surtout en anglais. La question de la violence était alors peu présente dans les préoccupations des associations féminines francophones.

La région urbaine d'Ottawa se dote en 1974 de services spécialisés dans la lutte contre la violence sexuelle : le Centre d'aide des victimes de viol d'Ottawa. Des intervenantes francophones y travaillent, les services s'affichent comme bilingues mais dans les faits, tous les quarts de travail ne sont pas comblés et l'ensemble des activités ne sont pas accessibles en français. Une des principales activités de sensibilisation et de dénonciation de la violence faite aux femmes est l'activité annuelle de la marche la Rue, la nuit, les femmes sans peur. Ces marches annuelles qui prennent racine dans des manifestations américaines contre la pornographie, réunissent de nombreuses femmes à partir de 1977 dans la région d'Ottawa-Carleton.

Par contre, dès le début des années 80 , avec l'émergence d'une identité féministe mieux assumée, les femmes francophones tenteront de se connaître et de se reconnaître. L'Union culturelle des femmes franco-ontariennes (UCFO) prend une place très 
importante sur l'échiquier de la francophonie féminine dans le domaine de la violence faite aux femmes et, particulièrement, de la violence sexuelle vécue dans l'enfance. L'UCFO met sur pied une campagne de sensibilisation sur l'abus sexuel dans l'enfance. Débutant en 1988 grâce à une subvention de la Direction générale de la condition féminine de l'Ontario, ce projet sera l'une des nombreuses activités touchant cette problématique au cours des cinq (5) années subséquentes. Les deux premières années sont consacrées à la sensibilisation des jeunes des écoles secondaires sur l'abus sexuel par la présentation d'une pièce de théâtre, Interdits. D'autre part, l'UCFO s'investit et prépare une vaste enquête sur la question de l'abus sexuel et les stratégies de survie des survivantes intitulée J'accepte de t'en parler. En 1993, elle prépare une vidéo, La lumière des mots, qui jette un éclairage sur la question du dévoilement de l'inceste en plus d'offrir une analyse très stimulante de cette problématique. En effet, cette vidéo présente des témoignages de femmes francophones de toutes les régions de l'Ontario qui ont subi diverses formes de sévices sexuels durant leur enfance et qui mettent en relief les conséquences de ces abus sur leur vie de même que leurs stratégies de survie. L'écoute, mais aussi la présence de groupes de soutien de femmes sont des éléments essentiels à leur guérison (Dupuis 1996 : 167). Les témoignages des femmes francophones sont importants pour se donner des lieux de parole, pour se reconnaitre. Ils contribuent à renforcer une identité féministe francophone.

Fin de la décennie 80, la question de l'application de la loi 8 est au coeur des débats. Ainsi, les participantes du colloque qui a donné lieu à la fondation d'Action ontarienne contre la violence faite aux femmes (AOcVF) en 1988 discuteront longuement de la création de services en français dans toutes les régions désignées. Leurs priorités de départ sont claires : réseautage et concertation (Drolet 1989 :6). Le colloque provincial de fondation de l'AOcVF, sera suivi en octobre 1994 par un colloque sur l'agression sexuelle. Ces colloques sont, en fait, des moments privilégiés pour faire le point sur les actions entreprises dans les dernières années, mais aussi des moments importants pour se rencontrer entre femmes francophones de l'Ontario. Le dernier colloque sera une occasion 
unique offerte aux femmes francophones qu'elles soient bénévoles, militantes, intervenantes, survivantes ou simplement intéressées par le sujet de l'agression sexuelle, de se concerter pour agir contre les multiples formes de violence faite aux femmes. Souvent précédés de conférences thématiques et de plénières, les ateliers de colloque sont autant de moments privilégiés pour s'informer que de lieux de discussion et de définitions des stratégies d'action.

La communauté féminine francophone a depuis longtemps fait preuve de concertation entre ses ressources, mais la décennie 90 verra la création d'un réseau spécialisé avec l'Action-ontarienne contre la violence faite aux femmes de même que l'opérationnalisation de la concertation dans l'ensemble des dossiers de condition féminine avec la création de la Table féministe de l'Ontario. Dans ce contexte, le mandat politique d'associations à vocation provinciale telles l'UCFO et la FFCF de l'Ontario se transforme, devenant membres d'un réseau. Tout en assumant toujours des responsabilités plus directes de formation et de sensibilisation auprès de leurs membres, leur volet politique se transforme et se démocratise en dehors des limites de leur vie associative ${ }^{5}$.

Par contre, les centres d'aide aux victimes d'agressions à caractère sexuel, créés au cours des années 70 dans plusieurs régions de l'Ontario, font défaut aux francophones, jusqu'à la décennie 90 , qui voit poindre des changements réels : la reconnaissance de leurs besoins spécifiques et sa matérialisation dans des ressources conçues par et pour elles. Ainsi dans l'Est ontarien, le rapport préparé par la Coopérative Convergence, Rendre visible l'invisible. Étude de besoins visant la création de services en français à l'intention des survivantes d'agressions à caractère sexuel résidant dans la région de la capitale nationale, met en évidence les lacunes. Leurs besoins les plus importants, de protection, de validation, de soutien et d'information, ne sont pas comblés. Où qu'elles se situent dans le continuum des besoins, les femmes francophones ont autant de difficulté à recevoir des services dans leur langue. Elles sont aussi marginalisées. Ce rapport donnera naissance au premier service exclusivement en français pour les femmes victimes d'agression à caractère sexuel de la région d'Ottawa-Carleton : le Centre francophone d'aide et de lutte pour mettre fin à la violence à 
caractère sexuel d'Ottawa-Carleton (CALACS). Il y a aussi un autre centre dans l'Est, bilingue celui-ci, le Service d'aide aux survivantes d'agression sexuelle de Stromont, Dundas, Glengarry et Akwesasne.

Le centreVictoria pour femmes, à Sudbury, est né aussi suite à une étude sur les besoins des femmes francophones de la région. Tout comme pour le CALACS francophone d'Ottawa, cette étude a été financée par le ministère du Solliciteur général et des Services correctionnels (Pharand 1996) afin de remplir leurs obligations liées à l'implantation de la loi 8 sur les services en français. Aussi dans le Nord, le Centre de Timmins est à dominante francophone. Dans le Sud, Oasis, Centre des femmes, est la seule ressource exclusivement francophone pour desservir la population du Toronto métropolitain. Créé en 1995 afin d'assurer aux femmes francophones des services touchant les agressions à caractère sexuel, il propose des cours d'auto-défense, des services directs auprès des femmes ayant vécu diverses formes d'agression dont des services d'accompagnement socio-judiciaire, des groupes de soutien, des services individualisés et des programmes de prévention dont Espace (Penwill 1996 : 178-180). Ailleurs, dans les autres sous-régions comme pour les comtés de Chatham et Kent, le Centre d'aide et de lutte contre les agressions sexuelles offre des services partiellement en français tout comme à Niagara, Hamilton, Sarnia,Windsor, Pembroke-Renfrew, Sault-Ste-Marie, North Bay.

\section{Des modèles féministes d'intervention en Ontario français, l'exemple de la problématique de l'agression sexuelle}

En Ontario francais, l'intervention féministe dans les situations d'agression à caractère sexuel est un modèle d'intervention intégré alliant intervention individuelle, intervention de groupe et intervention macro-collective. De plus, ce modèle intègre théorie/ pratique dans une réflexion féministe. Les CALACS de l'Ontario français ont développé un modèle d'intervention en s'inspirant très sensiblement de l'intervention radicale. Ils ont aussi adapté ce 
modèle à la réalité franco-ontarienne en l'inscrivant dans une analyse socio-politique des rapports sociaux de sexe dans le contexte franco-ontarien.

La situation des CALACS francophones de l'Ontario n'est pas sans rappeler l'expérience des CALACS québécois. Et comme le mentionne Vandal (1997), parmi les emprunts à d'autres approches, certains semblent avoir la faveur de plusieurs CALACS. C'est le cas de la thérapie radicale (Vandal 1997 :p.132). Parmi les concepts clefs de la thérapie radicale, mentionnons le concept d'oppression intériorisée ou le concept-flic (41).

Le travail en thérapie radicale consiste à détecter les messagesflics, les isoler du reste de la conscience, les dénoncer (en reconnaissant leurs sources externes), les déprogrammer et les reprogramer en faisant référence aux compétences et aux capacités de la personne elle-même. (Tremblay et Tremblay 1996 : 42).

Le concept central de la thérapie radicale est donc celui des oppressions intériorisées, communément appelées les flics, qui font partie de notre personnalité. Il s'agit de discours moralisteurs basés sur des idées fausses que nous avons intégrées à propos de nous-même. Le travail en thérapie radicale consiste à détecter les flics, à les isoler, à les dénoncer et à les combattre.

Dans les groupes de soutien du CALACS, un des objectifs est de travailler en coopération avec les personnes impliquées, en cherchant à établir les relations les plus égalitaires. Parmi les règles de coopération, trois sont essentielles, à savoir : « pas de secret, pas de mensonges; pas de sauvetage; pas de jeux de pouvoir " (42). Ainsi parmi ses outils de communication, la collective du CALACS francophone d'Ottawa s'est donné quelques règles touchant le sentiment retenu, l'impression-paranoia (pensée retenue) et les appréciations. Ces règles touchent aussi ses rapports avec la communauté et rappelons à cet égard, la soirée d'appréciation des bénévoles du CALACS, le 8 décembre 2001, pour fêter son $10^{\mathrm{e}}$ anniversaire. Un dernier outil emprunté à l'approche radicale comporte la nécessité de briser le triangle de protection (44). Ainsi, selon cette approche, le concept du triangle de sauvetage (ou triangle de protection) est utile "pour analyser les rôles joués 
par une personne dans ses relations (victime-sauveuse et persécutrice) »; le but est de briser cette dynamique (Vandal 1997:132).

Comme le précisait Strudiant (1990), l'approche féministe n'a pas fourni de nouvelles théories ou de nouvelles techniques d'intervention, mais une nouvelle façon de conceptualiser les problèmes vécus par les femmes.

Bien que les intervenantes des CALACS se trouvaient bien informées et conscientisées des tenants et aboutissants de l'analyse féministe de la problématique des agressions à caractère sexuel, elles n'étaient pas nécessairement suffisamment outillées pour faire face à la détresse vécue par les femmes et pour les aider à soulager celle-ci. L'analyse sociale et politique ne suffisait pas, il fallait trouver des moyens pour faire accéder les femmes à un plus grand bien-être (Vandal 1997 : 171).

L'approche radicale a été le vecteur de cette réflexion plus concrète.

Cependant, toutes les femmes francophones de l'Ontario n'ont pas accès à des services en français tels que ceux offerts dans les CALACS. L'exemple de concertation pour développer des services

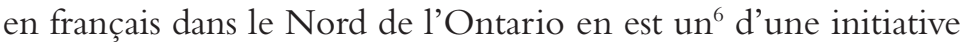
originale et créatrice. Deux priorités régionales ont été définies :

- la mise sur pied d'une ligne de crise régionale sans frais pour les femmes victimes d'agression sexuelle, de violence conjugale ou d'autres formes d'abus;

- le développement de services en matière de violence conjugale qui répondent efficacement aux besoins des femmes francophones de ces régions

(Dupont, Culligan et Pharand 2000 : 128)

À ce titre, depuis 1998, le CentreVictoria et le Centre Thérèse de Dubreuilville collaborent à développer des services en matière de violence faite aux femmes dans quelques communautés. Trois intervenantes communautaires sont présentes dans les communautés 
de Rayside-Balfour etVallée-Est (région de Sudbury), à Sault-Stemarie et à Dubreuilville (région d'Algoma).

Il s'agit donc d'un modèle de service qui s'inspire du principe de la toile d'araignée. Au lieu de concentrer les services dans un seul endroit où les femmes ont à se déplacer pour recevoir l'aide nécessaire, les intervenantes sont présentes dans la communauté, vont vers les femmes et s'adaptent à leurs besoins. (Dupont, Culligan et Pharand 2000 : 136-137)

Dans la région d'Ottawa, le Service familial catholique offre depuis des années des groupes de soutien pour les femmes survivantes d'agression à caractère sexuel. Les thématiques abordées dans les groupes s'articulent autour de quatre phases soit l'établissement d'un lieu sûr, briser l'isolement, la démarche de dévictimisation et finalement la réintégration et la conclusion du processus de chacune des participantes. Ces étapes sont empruntées à la démarche de Saxe (1993).

Parmi les outils d'intervention féministes utilisés dans les centres d'aide de l'Ontario, s'ajoutent à ceux offerts dans les CALACS québécois (groupe de support, suivi individuel, action politique), des cours d'autodéfense, la formation de bénévoles et des centres de documentation. Sur le plan plus pratique, la thérapie axée sur l'" insight ", les techniques cognitives, la thérapie brève et les groupes d'entraide sont considérés profitables aux femmes (Cholette 1996).Toutefois, Chantal Cholette rappelle « qu'aucun de ces modèles ne s'applique parfaitement à toutes les situations et que le choix du (ou des) modèle(s) varie selon l'orientation et l'approche de chaque centre" (Cholette 1996:4).

\section{L'intervention féministe : des intervenantes s'expriment}

Il appert que les fondements de l'intervention féministe sont toujours, trente ans après, au coeur du questionnement des intervenantes comme en font foi tant les publications des récentes 
années (De Koninck et Savard (1992)) que les témoignages des répondantes de l'enquête réalisée par Joanne Hart en 2001.

Cette cueillette des données, qui visait à recueillir les perspectives des intervenantes de diverses ressources communautaires de la région de l'Est de l'Ontario français ${ }^{7}$, s'est faite lors de trois (3) entrevues de groupe ${ }^{8}$. Ce type d'entrevue permet d'explorer les nuances et les complexités implicites aux attitudes et aux expériences des participantes (Hughes et DuMont 1993), dans ce cas-ci, les défis actuels de l'intervention féministe auprès des femmes ayant vécu l'agression sexuelle. De plus, il a été intéressant d'observer dans les groupes d'entrevue que le partage du vécu de chacune a permis l'approfondissement des perceptions des participantes en explorant les nuances et les complexités de leurs attitudes et de leurs expériences (Morgan et Spanish 1984; Morgan 1988; Hughes et DuMont 1993).

Six (6) intervenantes ${ }^{9}$ ont été recrutées pour participer au projet de recherche. D'après Hughes et DuMont (1993) (Mayer et St-Jacques 2000), ce nombre ${ }^{10}$ de participantes favorise l'émergence d'échanges approfondis tout en accordant suffisamment de temps à chacune des participantes pour explorer les thèmes à discuter. De ce fait, nous aurons eu recours à trois groupes d'entrevues, soit deux pour la cueillette des données et un troisième dans le but de valider les résultats de l'analyse du matériel recueilli précédemment. L'analyse du contenu de ces entretiens offre des pistes permettant d'élucider et d'approfondir les particularités de l'intervention féministe pratiquée en Ontario français.

\section{La permanence des fondements de l'intervention féministe}

L'exploration de deux des principes féministes, soit celui de favoriser des rapports plus égalitaires et celui de croire au potentiel des femmes, permet de constater qu'ils se chevauchent sur le plan de leur mise en pratique. Ainsi, toutes les intervenantes reconnaissent qu'il existe un certain déséquilibre entre l'intervenante et la cliente, déséquilibre que l'on ne peut nier. Cependant, en restant consciente de ce déséquilibre et en le nommant, il devient possible de le diminuer. Parmi les autres moyens utilisés 
pour démystifier le pouvoir personnel et favoriser des relations égalitaires, les répondantes laissent la femme "définir ses propres objectifs". Pour certaines, il est important de "valider ses connaissances, ses intuitions et de favoriser la reprise du pouvoir des femmes ». Pour d'autres, c'est de lui offrir un lieu où elle peut "juste être ». Enfin, une des répondantes a exprimé qu'elle accordait à la femme "le droit de me [l'intervenante] critiquer parce que je ne suis pas parfaite". De la même façon, cette répondante explique qu'une de ses responsabilités envers les femmes et l'organisme pour lequel elle travaille est de "clarifier son rôle " auprès de la femme et de lui fournir une compréhension de ce qu'elle peut et ne peut pas faire à l'intérieur de ce cadre d'intervention.

En échangeant sur les moyens pour démystifier le pouvoir professionnel et favoriser des rapports plus égalitaires, les intervenantes ont discuté longuement sur la notion de frontières. Les intervenantes reconnaissent que travailler avec une femme suscite parfois un «travail sur soi ». Dans de telles situations, certaines intervenantes vont partager ce qu'elles vivent avec la cliente dans la mesure où cette dernière peut profiter du partage. Cependant, toutes ont exprimé l'importance d'avoir un réseau de soutien de même que l'intérêt à " prendre soin de ce qui a été déclenché en elle pendant la rencontre ». Pour une des intervenantes, un autre moyen de travailler les limites ou les transferts et contre-transferts est d'utiliser le sentiment qui a été déclenché pendant la rencontre et de demander à la cliente si c'est ce qui se produit dans ses relations avec les membres de son entourage.

Certaines intervenantes traduisent le principe, croire au potentiel des femmes, en "honorant » le fait que la femme a survécu à la violence et ce, peu importe le mécanisme qu'elle a utilisé. Cela passe par la valorisation de ce que la femme "était avant l'agression et ce qu'elle sera après " tout comme par la capacité d'amener la femme à "s'émerveiller d'elle-même à partir de ses forces et ses capacités ». Ce principe est même pour l'une des répondantes, "l'énergie motrice " de son travail, une sorte de protection contre un burn-out. Enfin, ce principe s'étend à toute la durée de l'intervention, comme celui de reconnaitre aux femmes la capacité de prendre soin d'elles entre les rencontres. 


\section{Des outils féministes d'intervention}

Quant aux outils d'intervention féministe, la roue du pouvoir et les cercles développés par Ginette Larouche (1993) sont très utiles pour accompagner les femmes dans leur démarche. Une des participantes se sert de la roue du pouvoir pour aider la femme à identifier dans quelle sphère elle se retrouve et dans quelle sphère l'agresseur se situe. Pour cette dernière, la roue du pouvoir a l'avantage de démystifier certains éléments de la violence en plus de nommer toutes les formes de violence dont la femme peut avoir été victime. Une autre participante a mentionné se servir des cercles d'impuissance, de victimisation et de pouvoir dans le but d'expliquer aux femmes comment " on arrive à travers un conditionnement social à intérioriser certains messages et certaines réactions pour enfin arriver à porter seules les impacts de ceux-ci ». Enfin, les outils développés dans la formation en dévictimisation intitulée les mensonges qu'Horrore me comptait du Regroupement provincial des maisons d'hébergement et de transition pour femmes victimes de violence conjugale (Québec 1994) permettent d'identifier les messages sociaux intégrés par les femmes pour ensuite les amener à "se raconter une autre histoire " qui correspond davantage à leurs désirs et à leurs besoins.

Plusieurs intervenantes travaillent aussi à partir de ce qu'elles nomment l'auto-intervention. Par exemple, les intervenantes demandent aux femmes de prendre conscience de certains comportements ou réactions entre leurs rencontres. Le but de cet outil est de "rebrancher " la femme à chacun de "ses corps ", c'est-à-dire à son corps physique, spirituel et intellectuel. Le collage, le récit et le dessin sont aussi des outils que les intervenantes utilisent pour aider les femmes à cheminer. L'objectif de ces derniers est avant tout que la femme "fasse $d u$ sens " de son expérience et qu'elle puisse se "reconstruire ». Tel que souligné par une des intervenantes, faire un dessin donne du pouvoir aux femmes puisqu'elles sont maîtresses de leur dessin. Une autre participante croit qu'il peut être plus facile pour certaines femmes de dévoiler la violence qu'elles ont vécue par le dessin que verbalement, "c'est un autre moyen de lui donner une voix ». En 
dernier lieu, la respiration et la visualisation sont utilisées pour ancrer les femmes dans leurs expériences.

Pour terminer, bien que les outils d'intervention soient nombreux et très certainement utiles pour accompagner les femmes dans leur démarche, les participantes expriment tout de même que la formation continue leur permet de s'adapter aux nouvelles réalités des femmes. Par exemple, une intervenante a découvert récemment que la thérapie par le mouvement peut être une façon pour une femme de prendre sa place et de créer son espace personnel.

\section{Intervention féministe, travail professionnel et diversité sociale et culturelle}

Les défis auxquels les intervenantes sont confrontées sont nombreux, à savoir : la méfiance des ressources face à l'intervention féministe, l'adaptation difficile du modèle auprès des femmes de différentes communautés culturelles de même qu'auprès des femmes ayant des problèmes de santé mentale, la gestion de la colère et la violence portée par les femmes elles-mêmes. Ces divers aspects constituent les principaux éléments de leur réflexion.

Ainsi, les intervenantes ont noté que l'approche féministe a encore besoin d'être démystifiée puisqu'elle était «encore porteuse de mythes et de préjugés ". Ainsi comme la crédibilité de cette approche est souvent mise en cause, plusieurs croient que certaines ressources ne leur réferent pas les femmes.

D'autre part, certains principes de l'approche féministe «s'appliquent difficilement aux femmes de différentes cultures». Ainsi, puisque certaines de ces femmes n'ont que peu de droits civils et civiques dans leur société d'origine, elles semblent davantage désorganisées lorsque l'intervenante leur présente ses options. À ce sujet, le Regroupement québécois des CALACS reconnait aussi que les femmes immigrantes peuvent se sentir mal à l'aise dans une ressource comme un centre d'aide puisqu'elles vivent des réalités différentes des femmes d'ici et que l'intervention dans les CALACS est basée sur la réalité des femmes d'ici (Vandal 
1997). Conséquemment, il appartient aux intervenantes d'adapter leur intervention le plus possible à la réalité de toutes les femmes peu importe la culture. Une attitude d'humilité et d'ouverture est alors nécessaire pour reconnaître la diversité culturelle des femmes sans compter qu'il est difficile d'exprimer ses émotions dans une langue autre que sa langue maternelle (Vandal 1997).

Certaines intervenantes ont aussi mentionné qu'il était difficile "de vendre le pouvoir aux femmes" lorsqu'un problème de santé mentale empêche une femme de faire des choix. Ce défi se vit alors sur deux plans pour les intervenantes. Premièrement, elles sont confrontées aux conflits d'approches et de perspectives d'intervention différentes préconisées dans les diverses ressources de santé mentale. Deuxièmement, les intervenantes sont confrontées à leurs propres limites. Devant ces mêmes préoccupations, dans de telles situations, les CALACS ont opté pour considérer les femmes dans leur globalité, respecter leur rythme et leurs limites.

Travailler dans le domaine de la violence confronte aussi les intervenantes à leur propre vulnérabilité en tant que femmes. Elles se remettent en question sur ce qu'elles nomment le risque d'être "surmaternantes » envers les clientes. Elles portent en elles ce dilemme : autant il est quasi inévitable de leur offrir un peu de maternage, autant "par principe on ne devrait pas".

Dans le travail quotidien, le sens à donner à la colère des femmes lors des interventions est un enjeu réel.Ainsi, certaines répondantes des groupes d'entrevue éprouvent des difficultés avec l'expression de la colère. Une des participantes avoue parfois éprouver de la difficulté à distinguer entre les impacts de la violence et ce qui est devenu "sa peau " suite à la violence. Plus précisément, cette participante s'interroge à savoir "comment cadrer la colère qui est devenue un moyen de contrôle par rapport aux choses qu'une femme a intériorisées?" Pour une autre répondante, laisser exprimer la colère qui est fusionnée avec l'agresseur est un défi important puisque "lorsqu'elle est dans la phase où elle ressent l'agresseur dans l'âme, elle n'est plus victime, elle devient l'agresseur ". À ce titre, et en accord avec les principes d'intervention féministe, Duval (1986) suggère quatre pistes d'intervention pour aider les femmes à 
s'approprier leur colère dans les situations d'agressions sexuelles. En premier lieu, l'intervenante aide la cliente à se réapproprier son identité. Ce faisant, l'intervenante accompagne la femme afin de reconnaitre et de légitimer ses réactions et lui permettre de les manifester. En deuxième lieu, il est important de parler et de nommer les situations d'injustice sociale auxquelles sont confrontées les femmes. En troisième lieu, se tenir debout encourage les femmes à être actives dans leur démarche de dénonciation. Et finalement, se tenir ensemble fait appel à une solidarité collective afin d'enrayer les agressions.

En dernier lieu, deux défis mentionnés brièvement ont trait à la violence des femmes face aux enfants et à la violence dans les couples de lesbiennes. Et sans avoir de réponse claire sur ces difficiles questions, assez nouvellement nommées dans les services féministes, l'intervention féministe n'est pas, en soi, incapable de se positionner. Ainsi, Lyne Bouchard (1998) soulève deux raisons pour développer un modèle d'intervention féministe adapté aux dynamiques dans les relations lesbiennes. Tout d'abord, il y a lieu de penser que la dépendance émotive est davantage présente dans les dynamiques de violence chez les lesbiennes. Ensuite, le contexte social de discrimination envers les lesbiennes peut favoriser la dynamique de dépendance associée à la violence.

\section{Intervention féministe, francophonie et pouvoir politique}

Plusieurs auteures ont tenté de démontrer la spécificité culturelle des francophones (Brunet et al. 1998; Cholette 1996; Coopérative Convergence 1992; Sirois 1999). Selon ces dernières, les intervenantes et les survivantes francophones se heurtent à des obstacles que leurs homologues anglophones ne connaissent et ne reconnaissent pas. Ayant comme objectif de dénoncer les obstacles que rencontrent les femmes francophones de par leur appartenance à un groupe minoritaire, en vue de faire reconnaittre les besoins spécifiques de celles-ci, les auteures de ces textes souhaitent un jour que les services offerts aux femmes francophones seront suffisants pour répondre aux besoins des femmes survivantes francophones. Pour l'instant, les défis des intervenantes féministes 
francophones sont encore nombreux et plusieurs de leurs besoins restent à combler.

Les intervenantes féministes francophones rencontrées sont à la fois confrontées aux obstacles qui découlent de leur approche d'intervention et à ceux liés à la non reconnaissance de leur culture.

On ne peut pas travailler en violence faite aux femmes au niveau francophone en mettant l'accent juste sur le cycle de la violence et la violence. On a la lutte francophone, on a l'autre forme d'oppression qui est une différence de nos consoeurs anglophones. Oui il $y$ a l'autre forme d'oppression plus directe avec le gouvernement Harris mais en plus de ça, nous autres on a une double lutte donc c'est exigeant à deux niveaux.

Le plus grand défi soulevé par les participantes des groupes d'entrevue par rapport au type de travail qu'elles font est la non reconnaissance de leur contribution et ce, tant au sein de la communauté que dans les différents paliers gouvernementaux et politiques. Cette vision est partagée par les intervenantes regroupées au sein d'Action ontarienne contre la violence faite aux femmes. Ainsi, les coupures budgétaires, les politiques d'assimilation et l'influence de la religion catholique sont parmi les facteurs qui contribuent à maintenir les femmes francophones isolées donc inconnues des services sociaux (Cholette 1996; Sirois 1999). Lorsqu'il y en a, les services offerts aux survivantes francophones sont fragmentés et les femmes ont plus souvent qu'autrement recours à plusieurs services indirects avant d'être dirigées à une intervenante qui travaille auprès des femmes ayant vécu des agressions à caractère sexuel (Coopérative Convergence 1992).

Les impacts de cette non reconnaissance sont significatifs pour les intervenantes francophones. En premier lieu, elles reçoivent moins de financement de la part des bailleurs de fonds. Selon une des participantes, le premier défi consiste à «faire reconnaître que les services francophones sont aussi et même égaux en termes de valeur que les services anglophones ". Mais comment y arriver lorsque les 
organismes offrant des services francophones n'ont pas les ressources financières et humaines nécessaires pour pouvoir répondre à la demande, en plus de développer des services (Coopérative Convergence 1992)? Les participantes déplorent être constamment comparées aux services offerts par leurs homologues anglophones. Une des participantes dénonce l'écart entre les exigences de travail chez les francophones et chez les anglophones. Son agence exige qu'elle anime trois fois plus de groupes par année que son homologue anglophone. Enfin, les répondantes déplorent que "les outils francophones sont traduits en anglais et non l'inverse ». Selon Sirois, ce dernier obstacle fait partie de ceux auxquels sont systématiquement confrontés les regroupements de femmes: "absence de traduction aux rencontres, documentation en anglais seulement, obligation de faire valoir par un petit nombre de participantes les besoins de toute la communauté francophone" (1999: 17).

Plusieurs intervenantes des groupes d'entrevue ont aussi mentionné qu'en plus d'assumer une charge de travail considérable, elles sont très sollicitées pour participer aux rencontres de différents comités, municipaux, régionaux ou même provinciaux. Ainsi, ces dernières qui préconisent le principe de redonner le pouvoir aux femmes ne sont pas toujours à l'aise avec le fait qu'elles soient obligées de prendre le pouvoir en étant «LA » représentante de la communauté francophone. Une des répondantes des groupes d'entrevue décrit son malaise de la façon suivante : «En tant que féministe francophone, je veux redonner le pouvoir aux femmes, mais je m'impose de le prendre parce que si je le prends pas, qui va le prendre?". Mais participer à de tels comités en tant que représentante francophone donne aux femmes une visibilité qui pourrait signifier aussi une fragilité en termes de présence sur la place publique.

Moi je suis pour nommer [la discrimination], mais quand tu es toute seule de francophone dans le comité, tu n'oses pas nommer parce que tu te dis "là je m'embarque dans quoi? " Puis si je prends le risque, est-ce qu'ils vont encore impliquer au moins un peu les francophones? 
La question de l'isolement des femmes francophones est aussi au coeur d'une autre dynamique : les difficultés à rejoindre les femmes francophones et à leur offrir des services. Cette préoccupation est clairement identifiée parmi les intervenantes regroupées au sein d'Action ontarienne. Ainsi, parmi les raisons mises de l'avant pour expliquer cette difficulté, Lucie Brunet et al. (1998) mentionnent l'absence ou l'insuffisance de services francophones, les préjugés à l'égard des services francophones et l'assimilation des francophones. À ces raisons, les participantes des groupes d'entrevue ajoutent que les méthodes de recrutement des francophones ne sont pas valorisées. Une répondante explique qu' "on essaie de prendre les façons de faire des Anglais et des Anglaises puis ça ne marche pas ". Et une autre d'ajouter: "le recrutement des femmes francophones se fait complètement différemment que pour les femmes anglophones... c'est beaucoup plus du bouche à oreille".

Mais au coeur de la question de l'offre des services, se pose aussi celle de la visibilité des services en français sur la scène publique. Ainsi, une des intervenantes du groupe a souligné l'importance de s'identifier comme Franco-ontarienne afin de contrer les effets d'assimilation. Selon une des participantes oeuvrant dans un organisme bilingue, les francophones qui s'adressent en anglais à l'organisme où elle travaille se font référer aux services anglophones. De plus, plusieurs intervenantes francophones disent pouvoir compter sur les doigts de la main le nombre de références qu'elles ont reçues des organismes anglophones au cours des années. Ainsi, sans politique claire d'offre de services en français dans les services bilingues ou dans les services en général, les femmes ne sont pas référées automatiquement aux services en français. À cela, certains rétorquent que les femmes ne demandent pas de services en français. Mais ne faudrait-il pas aussi s'interroger sur les raisons qui poussent les femmes francophones à ne pas demander de services en français? En fait, pour Lucie Brunet et al. (1998), elles ne sont pas habituées à en recevoir et c'est sans compter que ces femmes sont probablement davantage préoccupées par leur propre survie qu'à revendiquer des services dans leur langue. 
Toutefois, pour celles qui utilisent les services en français liés à l'agression à caractère sexuel, les avantages sont nombreux. Et si plusieurs auteures reconnaissent les bienfaits d'entreprendre une démarche d'intervention dans sa langue maternelle (Cholette 1996; Sirois 1999), les répondantes de notre enquête notent que "quand on travaille dans notre langue maternelle, on travaille à beaucoup de niveaux. Si on travaille dans notre langue secondaire, on reste dans le rationnel parce qu'il faut y penser, ". Ainsi, la spécificité culturelle des francophones doit être reconnue pour développer de meilleurs outils d'intervention et créer de nouvelles ressources.

Par ailleurs, ces ressources doivent être ancrées dans l'expérience des femmes francophones de l'Ontario. Ainsi, la question du lien avec l'histoire des communautés francophones est un élément d'analyse incontournable dans le processus de guérison. Selon une des participantes:

"Si on décortique le processus de guérison d'une survivante et qu'on fait le lien avec l'historique des francophones, il est atteint à tous les niveaux. C'est là où notre spécificité a une origine dans qui on est, dans un contexte historique et culturel ».

D'après cette dernière, l'héritage culturel des femmes francophones est marqué par l'oppression et la victimisation et ce, tant sur le plan « de notre lien avec la religion, la spiritualité, la famille, notre droit à notre corps, notre sexualité, l'éducation, l'information... ». Une autre participante mentionne que la situation des francophones n'était pas reconnue dans les projets de multiculturalisme proposés par le gouvernement. L'obstacle principal pour les femmes francophones vient du fait qu'elles ne sont pas considérées au même titre qu'une autre minorité visible, «comme si les francophones n'avaient pas une identité qui leur était propre, ». Une autre participante a l'impression que le gouvernement a «entrouvert la porte à la francophonie pour être correct politiquement et non pas parce qu'il a compris ». 


\section{Conclusion}

Contre vents et marées, un mouvement francophone pour briser le silence sur les multiples facettes de la violence faite aux femmes et tentant de saisir l'identité culturelle des femmes francophones, est présent sur l'échiquier politique. Cette quête d'identité se greffe aussi à une tentative du mouvement des femmes francoontariennes de définition d'autres règles de pouvoir. En fait, comme le soulignent les chercheures dans le rapport Conditions de possibilités des services de santé et de services sociaux en français en Ontario : un enjeu pour les femmes, « les organismes de lutte contre la violence semblent avoir acquis une expérience politique et d'intervention qui pourrait servir de référence au développement de futures stratégies d'action ». (Andrew et al 1997)

Enfin, ce mouvement appartient à un mouvement international de lutte contre la violence faite aux femmes. Les marches La rue, la nuit, les femmes sans peur, les Cordes à linge $e^{11}$ et la Marche internationale des femmes qui se déroula à Ottawa en l'an 2000 donnent la couleur de ce mouvement. Tout comme celui-ci, il appartient à un vaste mouvement de revendications pour l'égalité et plus largement, les droits de la personne.

\section{Bibliographie}

Action ontarienne contre la violence faite aux femmes (AOcVF) (1998), Plan stratégique de développement des services en français en matière de violence contre les femmes, 1997-2001, document final septembre 1998. Ottawa, AOcVF.

Andrew, Caroline, Lyne Bouchard, Francois Boudreau, Linda Cardinal, Diane Farmer, Michèle Kérisit, Denise Lemire en collaboration avec Dyane Adam, Denise Cullingan, Hélène Dallaire, Anne Rochon-Ford (1997), Conditions de possibilités des services de santé et de services sociaux en français en Ontario : un enjeu pour les femmes, Ottawa, Table féministe francophone de concertation provinciale de l'Ontario.

Bouchard, Lyne (1998), Une violence doublement invisible. Quelques pistes de réflexion sur la violence dans les relations amoureuses entre lesbiennes, Ottawa, Collective lesbienne de l'Ontario.

Bourgon, Michèle (1987), "L'approche féministe en termes de rapports sociaux ou l'art de survivre sur la corde raide en talons hauts ", Service social, 36, 2-3; 248-273. 
Bourgon, Michèle et Christine Corbeil (1990), «Dix ans d'intervention féministe au Québec : bilan et perspectives ", Santé mentale au Québec, XV, $1: 205-222$.

Brunet, Lucie et al. (1998), Les services en français en matière de violence faite aux femmes : le défrichage d'un terrain tortueux, Profil de services en français en matière de violence contre les femmes, 1997-2001, Ottawa, $\mathrm{AOcVF}$.

Castro, Ginette, (1984), Radioscopie du féminisme américain, Parsi, Presses de la fondation nationale des sciences politiques.

Cholette, Chantal (1996), Baisser pavillon? Jamais! Manuel de formation à l'intention des intervenantes bénévoles travaillant à mettre fin à l'agression à caractère sexuel en Ontario français, Ottawa, La Coopérative Convergence.

Coopérative Convergence (1992), Rendre visible l'invisible. Étude de besoins visant la création des services en français à l'intention des survivantes d'agression à caractère sexuel résidant dans la région de la Capitale nationale, Ottawa, par Chantal Cholette et Lyne Bouchard.

Corbeil, Christine, Carole Lazure, Ginette Legault et Ann Paquet-Deehy (1983), L'intervention féministe :l'alternative des femmes au sexisme en thérapie, Montréal, Éditions St-Martin.

Côté, Andrée (1994), La guerre des sexes n'est pas finie. Notes sur l'évolution du droit canadien sur le viol, 1983-1993, Femmes d'action, vol 24, no $1: 24-33$.

De Koninck, Maria et Sylvie Savard (1992), De l'intervention féministe aux interventions féministes : des parcours multiples, une mémoire collective à construire, Rapport de recherche présenté au CQRS, Québec, Université Laval, Département de médecine sociale et préventive, doc. minéo.

Dupont, Diane, Denise Cullingan et Gaétane Pharand (2000), "Un travail de défrichage : le développement des services en matière de violence faite aux femmes dans le Nord de l'Ontario ", Reflets, Revue ontaroise d'intervention sociale et communautaire, vol 6, no 1 :128-140.

Dupuis, Nadya (1996), «L'Union culturelle des Franco-Ontariennes et la violence faite aux enfants », Reflets, Revue ontaroise d'intervention sociale et communautaire, vol 2, no $1: 166-168$.

Duval, Michèle (1986), "La nouvelle colère des femmes ", Atlantis, 12 : 1, 46-59.

Doré, Suzanne (1982), Les thérapies féministes, Conseil du statut de la femme du Québec, document de travail, doc. minéo.

Franks, Violet et Vasanti Burtle (dir) (1974), Women in Therapy, New Psychotherapies for a Changing Society, New-York, Brunner/Mazel Publishers.

Gaston, Louise et Sheilagh Hodgins (1984), «Les thérapies féministes : un nouveau système psychothérapeutique », Revue canadienne des sciences du comportements, 16,1:42-55.

Hughes, Diane et Kimberly DuMont (1993), «Using Focus Groups to Facilitate Culturally Anchored Research ", American Journal of Community Psychology, 21:6,pp 775-806.

Kirsh, Barbara (1974), "Consciousness-raising groups as therapy for women », Women in Therapy, New Psychotherapies for a Changing Society, New-York, Brunner/Mazel Publishers, 326-354.

Laing,Ronald 1967, The politics of Experience, New-York, Ballantine.

Laing (1971) L'équilibre mental, la folie et la famille, Paris, Maspero, traduit de l'anglais par Micheline Laguilmommie.

Lamoureux, Diane (1998), Les services féministes. De l'anti-étatisme à l'intégration subsidiaire dans Les femmes et l'État canadien, sous la direction de Manon Tremblay et Caroline Andrew, Ottawa, PUO. 
Lamoureux, Diane (1997), Féminisme et citoyenneté : sortir de l'ornière du féminin, Femmes et représentation politique au Québec et au Canada, Montréal, les Éditions du remue-ménage, Manon Tremblay et Caroline Andrew éd., 33-54.

Lamoureux, Diane (1990), «Les services féministes : de l'autonomie à l'extension de l'État-providence», Nouvelles pratiques sociales, 3,2:33-43.

Larouche, Ginette (1993), Aux formatrices en intervention auprès des femmes violentées, Montréal, Éditions Wilson et Lafleur.

Legault, Gisèle et Ann Pâquet-Deehy (1985), « Formation à l'intervention féministe à l'Université de Montréal ", Revue canadienne de service social, 274-282.

Levan, Andrea (1996),Violence Against Women in Janine Brodie (ed) Women and Canadian Public Policy, Toronto, Harcourt and Brace, 319-454.

Martin-Beausoleil, Geneviève (1982), «Intervention féministe et service social individualisé ", Service social, 31, 2-3; 308-329.

Mayer, Robert et Christine St-Jacques (2000), «L'entrevue de recherche, dans l'ouvrage » Méthodes de recherche en intervention sociale, sous la direction de Mayer et al. Montréal, Éditions Gaetan Morin, 287-325.

Morgan, David (1988) Focus Groups as Qualitative Research, Londres, Éditions Sage.

Morgan, David L. et Margaret T. Spanish (1984), "Focus groups ;A New Toll for Qualitative Research », Qualitative Sociology, 7-3:253-270.

Morgan, Robin (1970), Sisterhood is Powerful, New York, Randow House.

Paquet-Deehy, Ann, Maureen Dennie et Joane Turgeon (1992), L'intervention féministe prend racine et porte fruit. Recension des écrits de 1982-1992, Montréal, École de service social, Université de Montréal.

Penwill, Kathryn (1996), Oasis : Centre des femmes, Reflets, Revue ontaroise d'intervention sociale et communautaire, vol 2, no $1: 178-180$.

Pharand, Gaétane (1996), À l'aide des femmes francophones qui luttent contre les agressions à caractère sexuel, Reflets, Revue ontaroise d'intervention sociale et communautaire, vol 2, no 1: 101-115.

Sirois, Ghislaine (1999), Y a-t-il oppression des femmes francophones? Ottawa, AOcVF.

Tremblay, Danièle et Moïsette Tremblay (1996), "Le groupe en thérapie radicale, un outil pour redonner du pouvoir ", Intervention, $102: 39-47$.

Ursel, Jane (1994), EliminatingViolence Against Women : Reform or Co-optation in State Institutions, Power and resistance : Critical thinking About Canadian Social Issues, Samuelson L. (ed), Halifax, Fernwood Publishing.

Vandal, Claudette (1997), L'intervention féministe dans les centres d'aide et de lutte contre les agressions à caractère sexuel (CALACS) du Québec, Québec, Regroupement québécois des centres d'aide et de lutte contre les agressions à caractère sexuel.

\section{Notes}

1. Joanne Hart a reçu la bourse d'études du Regroupement des intervenantes et des intervenants contre les agressions sexuelles (Rifas); cette bourse vise à promouvoir la recherche portant sur la 
violence dans le cadre d'un mémoire de maitrise de l'École de service social de l'Université d'Ottawa.

2. Les entrevues ont été réalisées au printemps 2001 par Joanne Hart dans le cadre de la rechercheintervention en vue de l'obtention d'une maîtrise en service social à l'Université d'Ottawa, sous la direction de Cécile Coderre.

3. La thérapie radicale a surgi dans les années 60-70 à Berkeley en Californie. Cette approche a émergé de la réflexion d'individus appartenant à différents mouvements : féministes, intellectuels de gauche, anti-psychiatriques. (...) Il s'est inspiré des réflexions de Ronald Laing, Herbert Marcuse, Wilhelm Reich et Karl Marx. Il a remis en question l'organisation sociale partriarcale et capitaliste qui valorise la production et les rapports de force et de domination et qui classe les gens selon leur âge, sexe, couleur, rang social, orientation sexuelle etc. (Tremblay et Tremblay $1996: 40)$.

4. Ce terme est celui privilégié par le premier ouvrage en français au début de la décennie 80, ouvrage de référence encore très utilisé comme en témoignent les répondantes de l'enquête : Corbeil, Christine, Carole Lazure, Ginette Legault et Ann Paquet-Deehy (1983), L'intervention féministe : l'alternative des femmes au sexisme en thérapie, Montréal, Éditions St-Martin.

5. Par exemple, dès 1989 , l'UCFO fait partie d'un réseau d'intervenantes franco-ontariennes qui se penchent sur la question de la violence faite aux femmes. Et la FFCF de l'Ontario s'impliquera au milieu des années 90 dans la mise sur pied du Centre d'aide pour les femmes francophones victimes d'agressions et d'abus sexuel dans la région de Sudbury, le Centre Victoria.

6. Ce projet est issu du plan stratégique de développement des services en français en matière de violence contre les femmes (1997-2001) d'Action ontarienne contre la violence faite aux femmes (AOcVF 1998).

7. Pour des raisons de confidentialité, nous ne pouvons identifier les différentes ressources communautaires de la région qui nous ont partagé leurs expériences.

8. Entendons ici que cette méthode réfêre à « un groupe de personnes réunies pour participer à un entretien collectif sur un sujet précis » (Mucchielli 1996 cité dans Mayer et Saint-Jacques 2000: 122).

9. Un formulaire de consentement a dû être approuvé par le comité d'éthique de la recherche en sciences sociales et humanités de l'Université d'Ottawa et ensuite signé par chacune des participantes.

10. Quant au nombre de participantes, Morgan (1988) mentionne qu'au niveau pratique il est plus rentable d'interviewer plusieurs participantes à la fois alors qu'au niveau substantif, de plus petits groupes impliquent une plus grande participation de la part de chacune des participantes.

11. L'origine du Projet Cordes à linge remonte au début de la décennie alors que le Cape Cod Women's Agenda cherchait un moyen de sensibiliser le public au problème de la violence faite aux femmes. "Une artiste du groupe proposa de créer un étalage sur une corde à linge, de chandails dessinés par des survivantes. En plus de rendre compte du silence des femmes, les maillots flottant au vent lancent un message de protestation, de sensibilisation et surtout de réconciliation ". (Le Droit, le 17 mai 1999:8 par Patrice Gaudreault). En 1999, le Centre d'action des femmes contre la violence propose et coordonne la réalisation de ce projet Cordes à linge dans la région d'Ottawa. 